\title{
Improved model predictive control for three-phase Vienna rectifiers
}

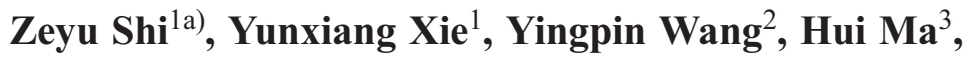 \\ and Jienan Zhang ${ }^{1}$ \\ ${ }^{1}$ School of Electric Power, South China University of Technology, \\ 381 Wushan Road, Guangzhou 510641, China \\ ${ }^{2}$ College of Physics \& Electronic Engineering, Hainan Normal University, \\ 99 Longkunnan Road, Haikou 571158, China \\ ${ }^{3}$ College of Electrical Engineering \& New Energy, China Three Gorges University, \\ 8 Daxue Road, Yichang 443002, China
}

a) zeyushi@163.com

\begin{abstract}
Vienna rectifiers are unidirectional three-level boost rectifiers used in extensive industrial applications. Much effort has been devoted to study the control strategies for Vienna rectifier. This paper introduces the manipulation principles and operation steps of model predictive control strategy based on optimal switching sequence principle. In order to address several issues of the conventional methods, an improved model predictive control strategy has been proposed to regulate the ac currents and neutral point voltage deviation. The proposed strategy has been validated by experiments. The consequences show that the proposed strategy can achieve accurate prediction especially during neutral point voltage unbalance by dealing with constraints and provide excellent performance in dynamic property and stability.
\end{abstract}

Keywords: model predictive control, three-phase Vienna rectifiers, control strategies, neutral point voltage balance

Classification: Power devices and circuits

\section{References}

[1] J. W. Kolar and F. C. Zach: "A novel three-phase utility interface minimizing line current harmonics of high-power telecommunications rectifier modules," IEEE Trans. Ind. Electron. 44 (1997) 456 (DOI: 10.1109/41.605619).

[2] M. Hartmann, et al.: "Digital current controller for a $1 \mathrm{MHz}, 10 \mathrm{~kW}$ three-phase Vienna rectifier,” IEEE Trans. Power Electron. 24 (2009) 2496 (DOI: 10.1109/ TPEL.2009.2031437).

[3] C. Qiao and K. M. Smedley: "Three-phase unity-power-factor star-connected switch (Vienna) rectifier with unified constant-frequency integration control," IEEE Trans. Power Electron. 18 (2003) 952 (DOI: 10.1109/TPEL.2003. 813759).

[4] L. Hang, et al:: "Digitized feedforward compensation method for high-powerdensity three-phase Vienna PFC converter," IEEE Trans. Ind. Electron. 60 (2013) 1512 (DOI: 10.1109/TIE.2012.2222851). 
[5] P. Cortes, et al:: "Predictive control in power electronics and drives," IEEE Trans. Ind. Electron. 55 (2008) 4312 (DOI: 10.1109/TIE.2008.2007480).

[6] S. Kouro, et al.: "Model predictive control—a simple and powerful method to control power converters," IEEE Trans. Ind. Electron. 56 (2009) 1826 (DOI: 10.1109/TIE.2008.2008349).

[7] J. Rodriguez, et al.: "Predictive current control of a voltage source inverter," IEEE Trans. Ind. Electron. 54 (2007) 495 (DOI: 10.1109/TIE.2006.888802).

[8] J. S. Lee and K. B. Lee: "Predictive control of Vienna rectifiers for PMSG systems,” IEEE Trans. Ind. Electron. 64 (2017) 2580 (DOI: 10.1109/TIE.2016. 2644599).

[9] S. Aurtenechea, et al:: "Predictive direct power control - a new control strategy for dc/ac converters," 32nd Annual Conference on IEEE Industrial Electronics (2006) 1661 (DOI: 10.1109/IECON.2006.347519).

[10] Y. Zhang, et al: "Model predictive direct power control of a PWM rectifier with duty cycle optimization," IEEE Trans. Power Electron. 28 (2013) 5343 (DOI: 10.1109/TPEL.2013.2243846).

[11] S. A. Larrinaga, et al.: "Predictive control strategy for dc/ac converters based on direct power control," IEEE Trans. Ind. Electron. 54 (2007) 1261 (DOI: 10. 1109/TIE.2007.893162).

[12] J. Hu and Z. Q. Zhu: "Improved voltage-vector sequences on dead-beat predictive direct power control of reversible three-phase grid-connected voltage-source converters," IEEE Trans. Power Electron. 28 (2013) 254 (DOI: 10.1109/TPEL.2012.2194512).

[13] S. Vazquez, et al:: "Predictive optimal switching sequence direct power control for grid-connected power converters," IEEE Trans. Ind. Electron. 62 (2015) 2010 (DOI: 10.1109/TIE.2014.2351378).

[14] Z. Song, et al.: "Predictive direct power control for three-phase grid-connected converters without sector information and voltage vector selection," IEEE Trans. Power Electron. 29 (2014) 5518 (DOI: 10.1109/TPEL.2013.2289982).

\section{Introduction}

Three-phase Vienna rectifiers have been widely used in industrial applications such as telecommunication systems, aircraft, power factor correction systems and wind turbine systems $[1,2]$ due to the advantages of compact size, low cost, high power density, high efficiency and low current total harmonic distortion (THD) [3, 4]. A lot of research has been carried out with respect to rectifier topologies, modulation strategies and control algorithms to achieve desired performance.

Model predictive control (MPC) has become a very attractive strategy for controlling power converters. Based on the discrete model, MPC can derive the prediction of control variables for future moments within finite prediction horizon. By minimizing the cost function evaluating the difference between prediction and reference, an optimal control sequence can be obtained. Depending on the property of the system input, MPC can be divided into finite control set (FCS) MPC and continuous control set (CCS) MPC. MPC is well known for its wide application ranges, fast dynamic response and capability of handling constraints [5, 6].

FCS-MPC is an intuitive and easy-to-implement MPC algorithm using the 
into the cost function. Search for the optimal control action minimizing the cost function's value [7]. The computational cost of FCS-MPC depends on the size of control set greatly. Therefore, the advantage of simplicity has been shown in Vienna rectifier for its less feasible switching patterns compared with neutral point clamped (NPC) three-level converter. However, FCS-MPC generates variable switching frequency and provides widespread harmonic spectrum [8].

The algorithm selecting the continuous control signals as the system input is referred to as CCS-MPC. Different from FCS-MPC, CCS-MPC provides constant switching frequency. Among various CCS-MPC, optimal switching sequence (OSS) MPC strategy considers the commutation instants as the system input. For the determined switching sequence, the optimal commutation instants achieving desired system behavior can be obtained by minimizing the cost function. In the strategy consisting of two vectors (a nonzero vector and a zero vector), the active vector can be selected by minimizing the cost function for all possible switching vectors similar to FCS-MPC $[9,10]$. A symmetrical switching sequence based on three voltage vectors was introduced in [11] in detail. In order to compensate the performance degradation caused by inappropriate switching sequence selection, several improved algorithms were presented in the literature. [12] redefined the candidate switching sequence in case of negative values of predicted duration times. A method avoiding the use of lookup tables was introduced in [13] by minimizing the cost function based on calculated optimal commutation instants for each sector in search of the global optimal switching sequence. [14] analyzed the relationship of duty cycles in each sector and proposed an algorithm without sector information of the grid voltage and the voltage vector selection process.

This paper proposed an improved model predictive control strategy for Vienna rectifiers based on optimal switching sequence principle. The constraints can not be addressed to achieve the optimal solutions by the conventional method. The proposed strategy takes the constraints into account when dealing with the optimal issues. The dynamic model of Vienna rectifier has been reconstructed. With the modified prediction, the minimal cost function evaluating the ac currents can be obtained. Additionally, the neutral point voltage difference has been introduced into the cost function. Compared with conventional method, the proposed strategy can obtain the accurate prediction of ac currents especially during neutral point voltage unbalance and system performance has been improved.

The rest of this paper is organized as follows. In Section 2, the conventional OSS-MPC strategy is introducing. In Section 3, the proposed OSS-MPC is presented in detail. In Section 4, experimental results are presented and analyzed. Finally, conclusions are given in Section 5.

\section{Conventional OSS-MPC strategy}

In this part, the conventional OSS-MPC strategy has been introduced. A lot of research was carried out in the literature. In order to achieve the desired performance, several important principles of OSS-MPC consists in discretized system model, prediction for control variables, the cost function evaluating the system outputs and minimizing algorithm deriving the optimal solutions. 


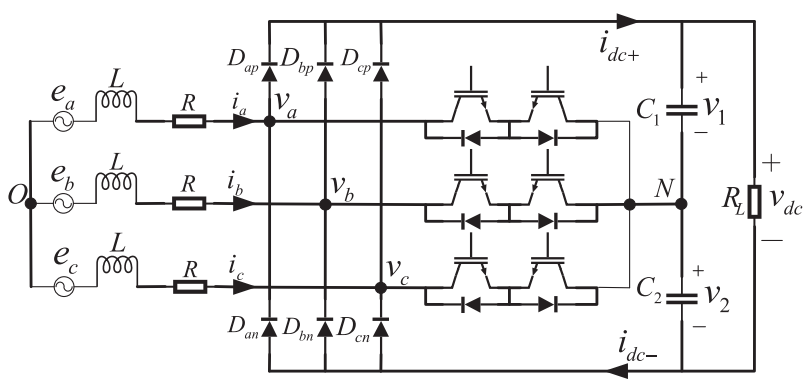

Fig. 1. Circuit diagram of Vienna rectifiers

\subsection{Model of Vienna rectifiers}

The circuit diagram of three-phase Vienna rectifiers is shown in Fig. 1. The rectifier is composed of a main diode bridge and three bidirectional switches connecting the input phases to the neutral point.

The continuous-time grid current dynamic of Vienna rectifier can be given as

$$
e_{i}=L \frac{d i_{i}}{d t}+R i_{i}+v_{i N}+v_{N O} \quad \forall i \in\{a, \mathrm{~b}, \mathrm{c}\}
$$

where $e_{i}$ and $i_{i}$ are grid voltages and input currents, respectively, $L$ and $R$ are the input filtering inductance and resistance, respectively, $v_{i N}$ is the switch voltage and $v_{N O}$ is the zero-sequence voltage.

If the bidirectional switch $S_{i}$ is on, $v_{i N}$ is clamped to zero and the corresponding phase current flows to the neutral point through bidirectional switch. If the bidirectional switch is off, $v_{i N}$ is determined by the direction of input current.

By analyzing the flow path of input currents, the currents through the positive and negative dc-bus can be obtained as

$$
\left\{\begin{array}{l}
i_{p}=S_{a p} i_{a}+S_{b p} i_{b}+S_{c p} i_{c} \\
i_{n}=S_{a n} i_{a}+S_{b n} i_{b}+S_{c n} i_{c}
\end{array}\right.
$$

where $S_{i p}$ and $S_{i n}$ denote the conducting state of up and down diode of each phase, respectively.

The dynamic model with respect to dc side of Vienna rectifier can be derived as

$$
\left\{\begin{array}{l}
C_{1} \frac{d v_{1}}{d t}=i_{p}-\frac{v_{1}+v_{2}}{R_{L}} \\
C_{2} \frac{d v_{2}}{d t}=i_{n}-\frac{v_{1}+v_{2}}{R_{L}}
\end{array}\right.
$$

where $C_{1}$ and $C_{2}$ are the dc side capacitances, and $R_{L}$ is the load resistance.

\subsection{Input currents prediction}

Based on the analysis in Section 2.1, there are three switching states in each phase as for the voltages between input phases and neutral point of dc-bus. Considering the different combination of three-phase switching states, 25 feasible switching states can be derived for Vienna rectifier. It is different from three-level neutral point clamped rectifier. Assuming that the utility grid is balanced and voltages of each dc side capacitor are equal, the voltages between input phases and neutral point of the grid can be obtained for each switching states. 
According to polarity of three-phase input currents, six sectors can be obtained. Due to the conducting characteristic of Vienna rectifier, the vector is feasible only when the polarity of its switching voltages is in accordance with the polarity of the corresponding phase currents. The operating ranges are constrained for Vienna rectifier. Based on the polarity of the phase current, the feasible vectors make up the hexagon in accordance with the determined sector.

Transform the dynamic model of Vienna rectifiers based on $d q$ synchronous rotating frame, Eq. (1) can be rewritten as

$$
\left\{\begin{array}{l}
\frac{d i_{d}}{d t}=\frac{1}{L}\left(e_{d}-R i_{d}-v_{d}+\omega L i_{q}\right) \\
\frac{d i_{q}}{d t}=\frac{1}{L}\left(e_{q}-R i_{q}-v_{q}-\omega L i_{d}\right)
\end{array}\right.
$$

The variation of input currents can be derived once the information of grid voltages, currents and switching voltages is determined. Define the variation of input currents as

$$
\left\{\begin{array}{l}
f_{d i}=\left.\frac{d i_{d}}{d t}\right|_{e=e_{k}, i=i_{k}, v=v_{i}} \quad \forall i \in\{1,2,3\} \\
f_{q i}=\left.\frac{d i_{q}}{d t}\right|_{e=e_{k}, i=i_{k}, v=v_{i}}
\end{array}\right.
$$

where $v_{i}$ is the switching voltages with $i \in\{1,2,3\}$.

Define the application times of vectors $v_{1}, v_{2}$ and $v_{3}$ as $t_{1}, t_{2}$ and $t_{3}$, respectively. With the knowledge of current variations and their application times, the prediction of grid current at the end of the sampling interval can be performed as

$$
\left\{\begin{array}{l}
\hat{i}_{d, k+1}=i_{d, k}+f_{d 1} t_{1}+f_{d 2} t_{2}+f_{d 3} t_{3} \\
\hat{i}_{q, k+1}=i_{q, k}+f_{q 1} t_{1}+f_{q 2} t_{2}+f_{q 3} t_{3}
\end{array}\right.
$$

\subsection{Cost function minimization}

The control objective of Vienna rectifier is to guarantee the input currents follow the reference accurately. To this end, it is essential to denote the error between the currents prediction and the reference. Thus, the quadratic cost function is introduced as

$$
J=\left(i_{d, k+1}^{*}-\hat{i}_{d, k+1}\right)^{2}+\left(i_{q, k+1}^{*}-\hat{i}_{q, k+1}\right)^{2}
$$

Substitute Eq. (6) into Eq. (7), $J$ is transformed into a quadratic function depending on $t_{1}, t_{2}$ and $t_{3}$ as

$$
J=\left(e_{d, k}-f_{d 1} t_{1}-f_{d 2} t_{2}-f_{d 3} t_{3}\right)^{2}+\left(e_{q, k}-f_{q 1} t_{1}-f_{q 2} t_{2}-f_{q 3} t_{3}\right)^{2}
$$

where $e_{d, k}=i_{d, k+1}^{*}-i_{d, k}$ and $e_{q, k}=i_{q, k+1}^{*}-i_{q, k}$.

In order to make the input currents follow the reference as exactly as possible, an optimal time set $\left\{t_{1}, t_{2}, t_{3}\right\}$ is determined to minimize cost function $J$.

The solution is found by solving the system as

$$
\left\{\begin{array}{l}
\frac{\partial J}{\partial t_{i}}=0 \\
t_{1}+t_{2}+t_{3}=t_{s}
\end{array} \quad \forall i \in\{1,2,3\}\right.
$$


The optimal time set $\left\{t_{1}, t_{2}, t_{3}\right\}$ minimizing the cost function $J$ corresponding to particular switching sequence is given as

$$
\left\{\begin{array}{l}
t_{1}=\frac{e_{d, k} f_{q 2}-e_{q, k} f_{p 2}-e_{d, k} f_{q 3}+e_{q, k} f_{p 3}+f_{p 2} f_{q 3} t_{s}-f_{p 3} f_{q 2} t_{s}}{f_{p 1} f_{q 2}-f_{p 2} f_{q 1}-f_{p 1} f_{q 3}+f_{p 3} f_{q 1}+f_{p 2} f_{q 3}-f_{p 3} f_{q 2}} \\
t_{2}=\frac{-e_{d, k} f_{q 1}+e_{q, k} f_{p 1}+e_{d, k} f_{q 3}-e_{q, k} f_{p 3}-f_{p 1} f_{q 3} t_{s}+f_{p 3} f_{q 1} t_{s}}{f_{p 1} f_{q 2}-f_{p 2} f_{q 1}-f_{p 1} f_{q 3}+f_{p 3} f_{q 1}+f_{p 2} f_{q 3}-f_{p 3} f_{q 2}} \\
t_{3}=t_{s}-t_{1}-t_{2}
\end{array}\right.
$$

For each vector sequence, the optimal time set can be calculated through Eq. (10) and with the specific application times, the value of cost function is derived. Among all sequences, the one minimizing the cost function is selected as optimal sequence and the corresponding time set is applied for the system.

\section{Proposed OSS-MPC strategy}

An improved OSS-MPC strategy has been proposed. Aiming at obtaining the constrained solutions considering all the constraints for application times, modification of the conventional strategy has been presented. Besides, issues of inaccurate prediction have been analyzed and an optimized prediction process has been proposed. Finally, the control target of neutral point voltage balance has been introduced into the control strategy.

\subsection{Constrained solutions for minimal cost function}

The research conclusion introduced in Section 2.3 is based on the assumption that the total application times are equal to the switching period. It is regarded as the constrained condition that must be observed.

Undesirably, there are situations that the optimal application times derived by Eq. (10) are negative and outside the feasible range during one sampling period.

Considering the practical operation, the application times must satisfy the following inequalities as $0 \leq t_{i} \leq t_{s}, i \in\{1,2,3\}$.

The equality constraints for Vienna rectifiers can be summarized as Table I.

In order to obtain the constrained solutions, the boundary conditions have to be analyzed. The constrained solutions exist if one or more component of application times is equal to 0 . Assume that the application times satisfy (2)(10) and constrained condition can be denoted as

$$
\left\{\begin{array}{l}
t_{1}+t_{2}=t_{s} \\
t_{3}=0
\end{array}\right.
$$

Substitute Eq. (11) into the cost function Eq. (8) and the cost function can be written as

Table I. Equality constraints of Vienna rectifiers 
Table II. Optimal solutions for each case

\begin{tabular}{|c|c|c|c|c|c|}
\hline Case & Constraints & Optimal Solution & Case & Constraints & Optimal Solution \\
\hline $\mathrm{A}$ & (1) & $(12)$ & $\mathrm{E}$ & (9) (10) & $\begin{cases}t_{s} & 0\end{cases}$ \\
\hline B & (2)(10) & $\left\{\left.t_{n}\right|_{n=1} \quad t_{s}-\left.t_{n}\right|_{n=1} \quad 0\right\}$ & $\mathrm{F}$ & (8)(10) & $\begin{cases}0 & t_{s} \\
\end{cases}$ \\
\hline $\mathrm{C}$ & (3)(9) & $\left\{t_{s}-\left.\left.t_{n}\right|_{n=3} \quad 0 \quad t_{n}\right|_{n=3}\right\}$ & G & (8) (9) & $\left\{\begin{array}{lll}0 & 0 & t_{s}\end{array}\right\}$ \\
\hline $\mathrm{D}$ & (4) 8 & $\left\{\begin{array}{ll}0 & \left.t_{n}\right|_{n=2} \quad t_{s}-\left.t_{n}\right|_{n=2}\end{array}\right\}$ & & & \\
\hline
\end{tabular}

$$
J^{\prime}=\left(e_{d, k}-f_{d 1} t_{1}-f_{d 2}\left(t_{s}-t_{1}\right)\right)^{2}+\left(e_{q, k}-f_{q 1} t_{1}-f_{q 2}\left(t_{s}-t_{1}\right)\right)^{2}
$$

Define

$$
t_{n}=\frac{\left(f_{p n}-f_{p(n+1)}\right)\left(e_{p, k}-f_{p(n+1)} t_{s}\right)+\left(f_{q n}-f_{q(n+1)}\right)\left(e_{q, k}-f_{q(n+1)} t_{s}\right)}{\left(f_{p n}-f_{p(n+1)}\right)^{2}+\left(f_{q n}-f_{q(n+1)}\right)^{2}}
$$

Make the derivative with respect to $t_{1}$ equal to 0 . The constrained solution can be derived from Table II as the result corresponding to Case B. Similarly, the optimal solutions corresponding to (3)(9) and (4)(8) can be obtained.

Taking each constrained condition into consideration, the optimal application times can be summarized as Table II. There are seven cases to be considered.

The minimal value of the cost function can be obtained by applying the solutions listed in Table II. Compare the results of Case A to G and search the optimal application times $\left\{t_{1}, t_{2}, t_{3}\right\}$ that have the minimal cost function.

\subsection{Prediction strategy for neutral voltage unbalance}

The prediction of $i_{d, k+1}$ and $i_{q, k+1}$ analyzed in Section 2.2 is based on the assumption that dc side voltages are balanced during the sampling interval, namely, $v_{1}=v_{2}=v_{d c} / 2$. However, due to the introduction of neutral point current $i_{o}$, the voltage error between two capacitors $C_{1}$ and $C_{2}$ is generated. It is inevitable to observe the voltage error and much effort is supposed to taken to decrease it. As a result, the variation of input currents during neutral point imbalance is different from that of balance condition. The nonzero error will make an impact on the prediction of input currents and the results made to follow the reference are not accurate compared with the actual input currents.

The current trajectories of $i_{d}$ and $i_{q}$ are shown in Fig. 2. The blue solid line and red solid line is the predicted currents when $v_{1}=v_{2}$ and $v_{1} \neq v_{2}$, respectively. $e_{i d}$ and $e_{i q}$ are the prediction error caused by neutral point voltage imbalance if the

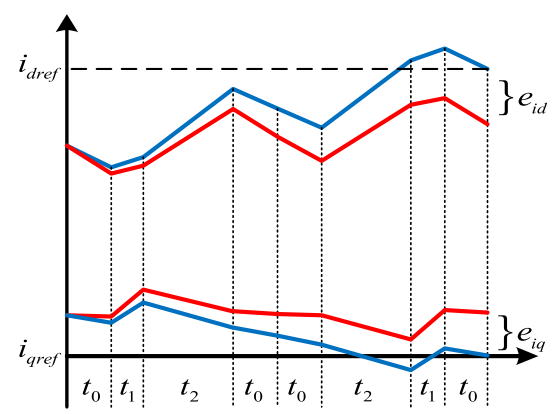

Fig. 2. Current trajectories of $i_{d}$ and $i_{q}$ 
Table III. Rectifier voltages $v_{\alpha}^{\prime}$ and $v_{\beta}^{\prime}$

\begin{tabular}{c|c|c|c|c|c}
\hline Vector & $v_{\alpha}^{\prime}$ & $v_{\beta}^{\prime}$ & Vector & $v_{\alpha}^{\prime}$ & $v_{\beta}^{\prime}$ \\
\hline$V_{1}$ & $2\left(v_{1}+v_{2}\right) / 3$ & 0 & $V_{06}$ & $v_{2} / 3$ & $-\sqrt{3} v_{2} / 3$ \\
\hline$V_{12}$ & $\left(2 v_{1}+v_{2}\right) / 3$ & $\sqrt{3} v_{2} / 3$ & $V_{61}$ & $\left(2 v_{1}+v_{2}\right) / 3$ & $-\sqrt{3} v_{2} / 3$ \\
\hline$V_{02}$ & $v_{2} / 3$ & $\sqrt{3} v_{2} / 3$ & \multirow{2}{*}{$V_{01}$} & $2 v_{1} / 3$ & 0 \\
\hline$V_{0}$ & 0 & 0 & & $2 v_{2} / 3$ & 0 \\
\hline
\end{tabular}

inaccurate predicted currents are forced to follow the reference. Modification of the prediction strategy is essential to improve the performance for Vienna rectifier.

Denoting $v_{i N}$ using $v_{1}$ and $v_{2}$ instead of $v_{d c}$ and considering system model Eq. (1), the rectifier voltages $v_{\alpha}^{\prime}$ and $v_{\beta}^{\prime}$ can be given in Table III.

It is noticeable that the results are identical to the conventional ones when $v_{1}=v_{2}=v_{d c} / 2$. In addition, there are two different vectors representing $V_{01}$, namely, the switching states describing small vectors provide distinct currents variations. The modified rectifier voltages can be illustrated in the $\alpha-\beta$ plain as Fig. 3.

Introduce the error of dc side two capacitors as

$$
\Delta v=v_{1}-v_{2}
$$

The modified currents variation $F_{j}^{\prime}=\left[\begin{array}{ll}f_{d j} & f_{q j}\end{array}\right]^{T}$ can be expressed as

$$
F_{j}^{\prime}=F_{j}+\frac{A}{L} E_{j} \Delta v
$$

where $A$ is coordinate transformation matrix transforming $v_{\alpha \beta}$ into $v_{d q}, L$ is input filtering inductance, $E_{j}$ is the error matrix expressed in Table IV and $\Delta v=v_{1}-v_{2}$ is the error of dc side two capacitors.

From Table III, it can be observed that the switching states representing small vectors have different current variations. It is necessary to assign the application times to them separately and the time set can be written as $\left\{t_{1}, t_{2}, t_{01}, t_{02}\right\} . t_{01}$ and $t_{02}$ represent the application times of switching states describing small vectors.

The predicted input currents can be rewritten as

$$
\left\{\begin{array}{l}
\hat{i}_{d, k+1}=i_{d, k}+f_{d 1}^{\prime} t_{1}+f_{d 2}^{\prime} t_{2}+f_{d 01}^{\prime} t_{01}+f_{d 02}^{\prime} t_{02} \\
\hat{i}_{q, k+1}=i_{q, k}+f_{q 1}^{\prime} t_{1}+f_{q 2}^{\prime} t_{2}+f_{q 01}^{\prime} t_{01}+f_{q 02}^{\prime} t_{02}
\end{array}\right.
$$

Apply the modified predicted input currents to the implementation system of OSSMPC strategy. The optimal vector sequence and application times can be obtained

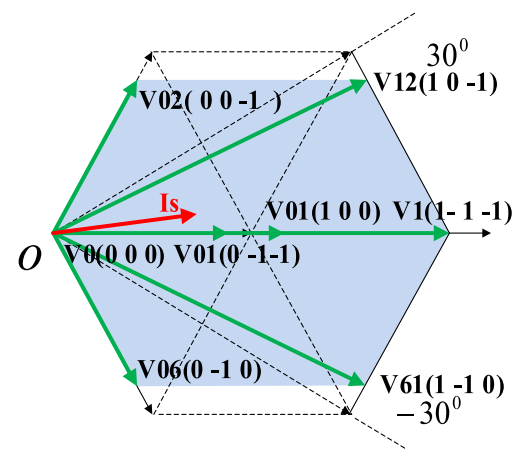

Fig. 3. Modified voltage vectors for sector I 
Table IV. Expression of $E_{j}$

\begin{tabular}{|c|c|c|c|}
\hline$j$ & $E_{j}$ & $j$ & $E_{j}$ \\
\hline$E_{1}$ & {$\left[\begin{array}{ll}0 & 0\end{array}\right]^{T}$} & $E_{06}$ & {$\left[\begin{array}{ll}-1 / 6 & \sqrt{3} / 6\end{array}\right]^{T}$} \\
\hline$E_{12}$ & {$\left[\begin{array}{ll}1 / 6 & -\sqrt{3} / 6\end{array}{ }^{T}\right.$} & $E_{61}$ & {$\left[\begin{array}{ll}1 / 6 & \sqrt{3} / 6\end{array}\right]^{T}$} \\
\hline$E_{02}$ & {$\left[\begin{array}{ll}-1 / 6 & -\sqrt{3} / 6\end{array}\right]^{T}$} & \multirow{2}{*}{$E_{01}$} & {$\left[\begin{array}{ll}1 / 3 & 0\end{array}\right]^{T}$} \\
\hline$E_{0}$ & {$\left[\begin{array}{ll}0 & 0\end{array}\right]^{T}$} & & {$\left[\begin{array}{ll}-1 / 3 & 0\end{array}\right]^{T}$} \\
\hline
\end{tabular}

by minimizing the cost function describing the error between prediction and reference.

\subsection{Neutral point voltage balance control}

Two capacitors composing the dc side of Vienna rectifier are analyzed to study the operating principle of neutral point voltage unbalance. The charge-discharge characteristics and equivalent impedances of capacitors influencing the neutral point voltage fluctuation can be determined by the manufacturing processes and stray parameters. In addition, the neutral point current containing ac fluctuation and dc offset components can lead to neutral point voltage unbalance.

Based on the circuit diagram of Vienna rectifiers, the mathematical model of two dc-link capacitors can be given as

$$
\left\{\begin{array}{l}
i_{C_{1}}=C_{1} \frac{d v_{1}}{d t} \\
i_{C_{2}}=C_{2} \frac{d v_{2}}{d t}
\end{array}\right.
$$

Assume that $C_{1}=C_{2}=C$ and integrate Eq. (17), the voltages of capacitors can be derived as

$$
\left\{\begin{array}{l}
v_{1, t_{2}}=\frac{1}{C} \int_{t_{1}}^{t_{2}} i_{C_{1}} d t+v_{1, t_{1}} \\
v_{2, t_{2}}=\frac{1}{C} \int_{t_{1}}^{t_{2}} i_{C_{2}} d t+v_{2, t_{1}}
\end{array}\right.
$$

Apply $t_{1}=t_{k+1}$ and $t_{2}=t_{k}$ to Eq. (18). The variation of capacitor voltages $\Delta v$ is expressed as

$$
\Delta v_{k+1}=\frac{1}{C} \int_{k}^{k+1}\left(i_{C_{1}}-i_{C_{2}}\right) d t+\Delta v_{k}
$$

The current difference of two capacitors related to the input currents and switching states can be expressed as

$$
i_{C_{1}}-i_{C_{2}}=-\left(i_{a} S_{a}+i_{b} S_{b}+i_{c} S_{c}\right)
$$

Assume the currents flowing through the capacitors are constant during the sampling interval and the variation of capacitor voltages can be derived as 


$$
\Delta v_{k+1}=-\frac{1}{C}\left(i_{a} S_{a}+i_{b} S_{b}+i_{c} S_{c}\right) t_{s}+\Delta v_{k}
$$

where $t_{s}$ is the sampling period.

Define a function that describe the variation of neutral point voltage difference as

$$
f_{v i}=\left.\frac{d \Delta v}{d t}\right|_{S_{a b c}=S_{a b c i}}=i_{a} S_{a i}+i_{b} S_{b i}+i_{c} S_{c i}
$$

The prediction of neutral point voltage difference at the end of the sampling period can be computed as

$$
\Delta \hat{v}_{k+1}=\Delta v_{k}+f_{n 1} t_{1}+f_{n 2} t_{2}+f_{n 01} t_{01}+f_{n 02} t_{02}
$$

To achieve neutral point voltage balance, the voltage deviation is set equal to zero. Introducing the weighting factor representing the adjustment between input currents and neutral point voltage, the cost function containing the quadratic difference between the prediction and reference for neutral point voltage can be given as

$$
J=\left(i_{d, k+1}^{*}-\hat{i}_{d, k+1}\right)^{2}+\left(i_{q, k+1}^{*}-\hat{i}_{q, k+1}\right)^{2}+\lambda\left(\Delta v_{k+1}^{*}-\Delta \hat{v}_{k+1}\right)^{2}
$$

Transform Eq. (24) into

$$
\begin{aligned}
J= & \left(e_{d, k}-f_{d 1} t_{1}-f_{d 2} t_{2}-f_{d 01} t_{01}-f_{d 02} t_{02}\right)^{2} \\
& +\left(e_{q, k}-f_{q 1} t_{1}-f_{q 2} t_{2}-f_{q 01} t_{01}-f_{q 02} t_{02}\right)^{2} \\
& +\lambda\left(e_{u, k}-f_{n 1} t_{1}-f_{n 2} t_{2}-f_{n 01} t_{01}-f_{n 02} t_{02}\right)^{2}
\end{aligned}
$$

where $e_{u, k}=\Delta v_{k+1}^{*}-\Delta v_{k}$.

Make the partial derivative of $J$ with respect to application times equal to zero, the optimal solution can be obtained as

$$
\left\{\begin{array}{l}
t_{1}=\frac{m_{11} e_{d, k}+m_{12} e_{q, k}+m_{13} e_{u, k}+m_{14} t_{s}}{m_{11} f_{p 1}+m_{12} f_{q 1}+m_{13} f_{n 1}+m_{14}} \\
t_{2}=\frac{m_{21} e_{d, k}+m_{22} e_{q, k}+m_{23} e_{u, k}+m_{24} t_{s}}{m_{21} f_{p 2}+m_{22} f_{q 2}+m_{23} f_{n 2}+m_{24}} \\
t_{01}=\frac{m_{31} e_{d, k}+m_{32} e_{q, k}+m_{33} e_{u, k}+m_{34} t_{s}}{m_{31} f_{p 01}+m_{32} f_{q 01}+m_{33} f_{n 01}+m_{34}} \\
t_{02}=\frac{m_{41} e_{d, k}+m_{42} e_{q, k}+m_{43} e_{u, k}+m_{44} t_{s}}{m_{41} f_{p 02}+m_{42} f_{q 02}+m_{43} f_{n 02}+m_{44}}
\end{array}\right.
$$

where polynomial $m_{i j}$ is expressed in Table V.

\section{Experimental results}

In order to validate the effectiveness of the theoretical derivation, experiments have been carried out based on the proposed strategy. The three-phase Vienna rectifier is shown in Fig. 1. The system parameters are listed in Table VI. The Vienna rectifier is controlled to operate in unity input power factor and the reactive power reference is set to zero. The control strategy has been coded according to the control process introduced in this paper based on the digital control platform consisting of TMS320F28335 DSP. In addition, the conventional model predictive control strategy is implemented to compare with the proposed strategy. 
Table V. Expression of polynomial $m_{i j}$

\begin{tabular}{|c|c|c|c|}
\hline$m_{i j}$ & Expression & $m_{i j}$ & Expression \\
\hline$m_{11}$ & $f_{n 01}\left(f_{q 02}-f_{q 2}\right)+f_{n 2}\left(f_{q 01}-f_{q 02}\right)+f_{n 02}\left(f_{q 2}-f_{q 01}\right)$ & $m_{31}$ & $f_{n 1}\left(f_{q 2}-f_{q 02}\right)+f_{n 2}\left(f_{q 02}-f_{q 1}\right)+f_{n 02}\left(f_{q 1}-f_{q 2}\right)$ \\
\hline$m_{12}$ & $f_{n 01}\left(f_{p 2}-f_{p 02}\right)+f_{n 02}\left(f_{p 01}-f_{p 2}\right)+f_{n 2}\left(f_{p 02}-f_{p 01}\right)$ & $m_{32}$ & $f_{n 1}\left(f_{d 02}-f_{p 2}\right)+f_{n 2}\left(f_{p 1}-f_{p 02}\right)+f_{n 02}\left(f_{p 2}-f_{p 1}\right)$ \\
\hline$m_{13}$ & $f_{p 01}\left(f_{q 2}-f_{q 02}\right)+f_{p 02}\left(f_{q 01}-f_{p 02}\right)+f_{p 2}\left(f_{q 02}-f_{q 01}\right)$ & $m_{33}$ & $f_{p 1}\left(f_{q 02}-f_{q 2}\right)+f_{p 2}\left(f_{q 1}-f_{q 02}\right)+f_{p 02}\left(f_{q 2}-f_{q 1}\right)$ \\
\hline$m_{14}$ & $\begin{array}{l}f_{n 01}\left(f_{p 02} f_{q 2}-f_{p 2} f_{q 02}\right)+f_{n 2}\left(f_{p 01} f_{q 02}-f_{p 02} f_{q 01}\right) \\
+f_{n 02}\left(f_{p 2} f_{q 01}-f_{p 01} f_{q 2}\right)\end{array}$ & $m_{34}$ & $\begin{array}{l}f_{n 1}\left(f_{p 2} f_{q 02}-f_{p 02} f_{q 2}\right)+f_{n 2}\left(f_{p 02} f_{q 1}-f_{p 1} f_{q 02}\right) \\
+f_{n 02}\left(f_{p 1} f_{q 2}-f_{p 2} f_{q 1}\right)\end{array}$ \\
\hline$m_{21}$ & $f_{n 01}\left(f_{q 1}-f_{q 02}\right)+f_{n 02}\left(f_{q 01}-f_{q 1}\right)+f_{n 1}\left(f_{q 02}-f_{q 01}\right)$ & $m_{41}$ & $f_{n 1}\left(f_{q 01}-f_{q 2}\right)+f_{n 2}\left(f_{q 1}-f_{q 01}\right)+f_{n 01}\left(f_{q 2}-f_{q 1}\right)$ \\
\hline$m_{22}$ & $f_{n 01}\left(f_{p 02}-f_{p 1}\right)+f_{n 02}\left(f_{p 1}-f_{p 01}\right)+f_{n 1}\left(f_{p 01}-f_{p 02}\right)$ & $m_{42}$ & $f_{n 1}\left(f_{p 2}-f_{p 01}\right)+f_{n 2}\left(f_{p 01}-f_{p 1}\right)+f_{n 01}\left(f_{p 1}-f_{p 2}\right)$ \\
\hline$m_{23}$ & $f_{p 01}\left(f_{q 02}-f_{q 1}\right)+f_{p 02}\left(f_{q 1}-f_{q 01}\right)+f_{p 1}\left(f_{q 01}-f_{q 02}\right)$ & $m_{43}$ & $f_{p 1}\left(f_{q 2}-f_{q 01}\right)+f_{p 2}\left(f_{q 01}-f_{q 1}\right)+f_{p 01}\left(f_{q 1}-f_{q 2}\right)$ \\
\hline$m_{24}$ & $\begin{array}{l}f_{n 01}\left(f_{p 1} f_{q 02}-f_{p 02} f_{q 1}\right)+f_{n 1}\left(f_{p 02} f_{q 01}-f_{p 01} f_{q 02}\right) \\
+f_{n 02}\left(f_{p 01} f_{q 1}-f_{p 1} f_{q 01}\right)\end{array}$ & $m_{44}$ & $\begin{array}{l}f_{n 01}\left(f_{p 2} f_{q 1}-f_{p 1} f_{q 2}\right)+f_{n 2}\left(f_{p 1} f_{q 01}-f_{p 01} f_{q 1}\right) \\
+f_{n 1}\left(f_{p 01} f_{q 2}-f_{p 2} f_{q 01}\right)\end{array}$ \\
\hline
\end{tabular}

Table VI. System parameters

\begin{tabular}{c|c|c}
\hline Symbol & Description & Value \\
\hline$e$ & Grid phase voltage & $110 \mathrm{~V}$ \\
\hline$f$ & Grid frequency & $50 \mathrm{~Hz}$ \\
\hline$L$ & Input filtering inductance & $3 \mathrm{mH}$ \\
\hline$C$ & Dc-link capacitance & $2200 \mathrm{uF}$ \\
\hline$v_{d c}$ & Dc-link voltage & $300 \mathrm{~V}$ \\
\hline$P$ & Rated output power & $2 \mathrm{~kW}$ \\
\hline$f_{s}$ & Switching frequency & $10 \mathrm{kHz}$ \\
\hline
\end{tabular}

In Fig. 4, the dynamic performance of conventional OSS-MPC and proposed OSS-MPC is depicted. The load change from no load to full load has been carried out. From the ac current waveforms obtained in the consequences, the proposed strategy has shown its superiority in dynamic response rapidity and less current overshoot can be achieved. As a result, the dynamic performance of load change can be improved by using the proposed OSS-MPC.

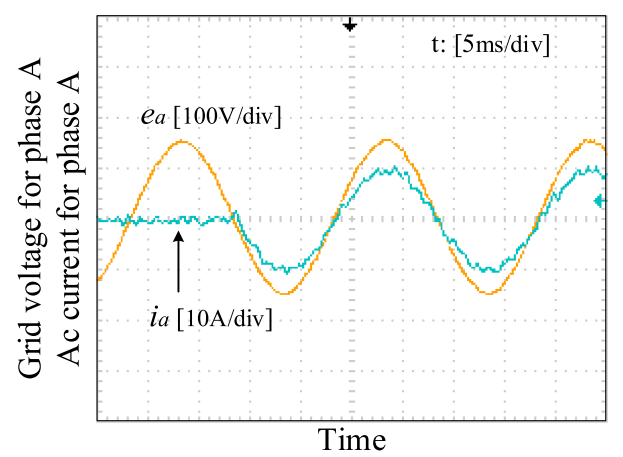

(a)

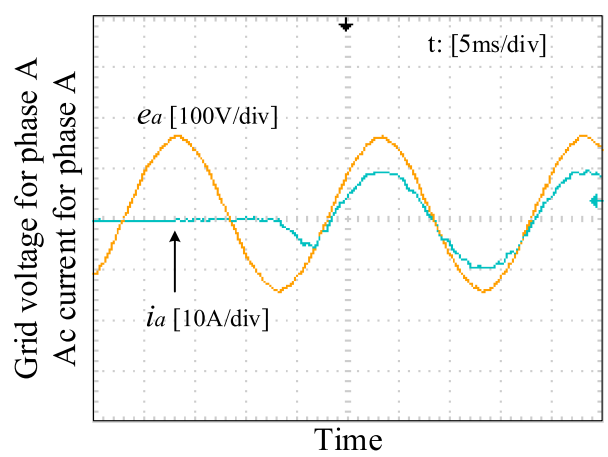

(b)

Fig. 4. System response to load change from no load to full load for (a) conventional OSS-MPC (b) proposed OSS-MPC 


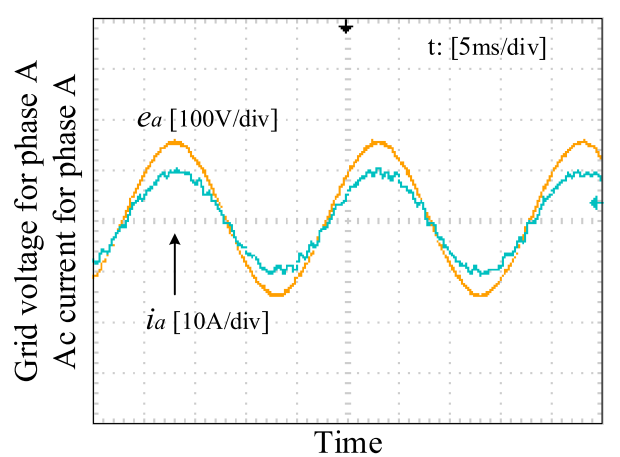

(a)

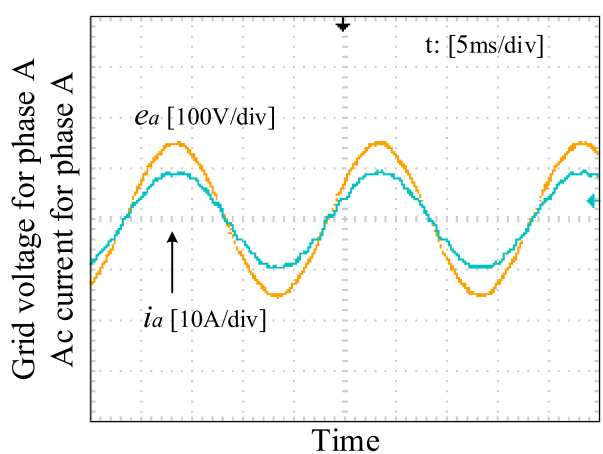

(b)

Fig. 5. Grid voltage and ac current of phase A during steady state for (a) conventional OSS-MPC and (b) proposed OSS-MPC

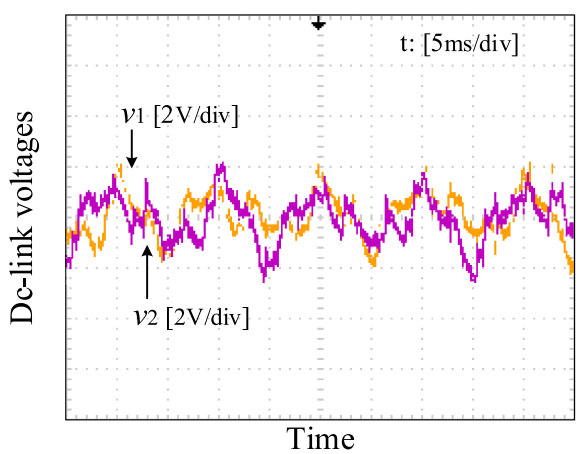

(a)

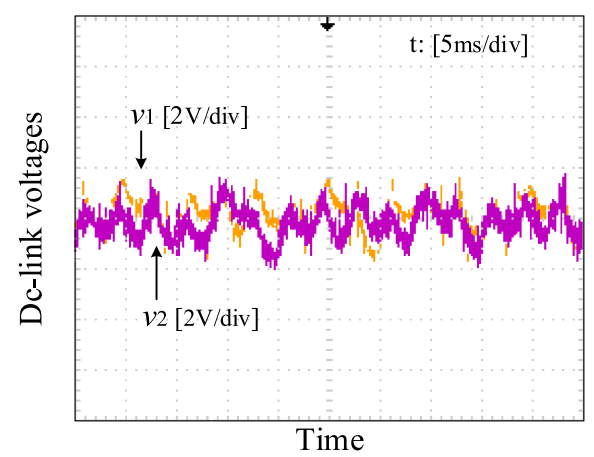

(b)

Fig. 6. Dc-link voltages during steady state for (a) conventional OSSMPC and (b) proposed OSS-MPC

has been improved by the proposed strategy deriving the THD of $4.1 \%$. It is noticeable that the proposed OSS-MPC can achieve desired sinusoidal phase currents and unity power factor. Fig. 6 shows the experimental waveforms for dc-link voltages during steady state. Compared the conventional OSS-MPC, dclink voltage ripple consisting of smaller amplitude can be achieved by the proposed OSS-MPC. As a result, the proposed OSS-MPC can reduce the dc-link voltage ripple and improve the steady state performance.

\section{Conclusion}

Model predictive control has attracted more and more attention for its good performance. However, during the prediction and optimization process, several issues are essentially needed to be addressed. In this paper, an improved model predictive control strategy has been presented following optimal switching sequence principle. Based on the analysis of the predicted dynamic model, accurate currents prediction and minimization have been carried out to improve the performance by using the proposed strategy. The experimental results have been carried out. As a result, the proposed strategy has shown the capability in practical applications for its effectiveness and prospective research values. 